\title{
Journal of Affective Disorders
}

BRIEF REPORT

\section{Deep brain stimulation of the posterior gyrus rectus region for treatment resistant depression}

Ettore A. Accolla ${ }^{a, b, f}$, Sabine Aust ${ }^{c}$, Angela Merkla, ${ }^{a, c}$, Gerd-Helde Schneider ${ }^{d}$, Andrea A. Kühn ${ }^{a}$, Malek Bajboujc, and Bogdan Draganski. ${ }^{\text {be }}$

aDepartment of Neurology, Charité University Medicine Berlin, Campus Virchow (CVK), 13353 Berlin, Germany.

bLREN - Département des neurosciences cliniques, CHUV, Université de Lausanne, 1011 Lausanne, Switzerland.

'Department of Psychiatry, Center for Affective Sciences (CAS), Charité and Freie Universität Berlin, Campus Benjamin Franklin (CBF), 14050 Berlin, Germany.

'Department of Neurology, Charité University Medicine Berlin, Campus Virchow (CVK), 13353 Berlin, Germany.

'Max Planck Institute for Human Cognitive and Brain Science, 04103 Leipzig, Germany.

${ }^{f}$ Neurology Unit, Department of Medicine, University of Fribourg, Fribourg, Switzerland (current address).

Word Count: Abstract: 267 words; Text 1942 words

\section{Corresponding Author :}

Bogdan Draganski

LREN, Département des Neurosciences Cliniques

CHUV, Université de Lausanne

Rue de Bugnon 21, CH-1011 Lausanne

Tel: +41213149593

Fax: +41 213141256

email: bogdan.draganski@gmail.com 


\section{Abstract}

Background: Deep brain stimulation (DBS) represents an alternative symptomatic treatment for major depressive disorder in case of failure of pharmacotherapy. The sub-genual cingulate - Brodmann area 25 (CG-25), is one of the most widely used targets for electrode implantation. Given the diverging clinical outcome after DBS, there is a pressing need for in-depth study of brain anatomy and function allowing accurate and reliable prognosis before surgery.

Methods: We studied five treatment-resistant major depressive disorder patients planned to undergo DBS targeting the CG-25. Before surgery, we acquired high-resolution magnetic resonance (MR) diffusion-weighted images for each patient followed by post-surgery MRI for electrode localization. To estimate individual anatomical connectivity pattern of the active contact location we performed probabilistic diffusion tractography intra-individually. We then correlated connectivity patterns with outcome assessed with standardized clinical tests. Connectivity results were compared between DBS responders and non-responders.

Results: We observed in one patient an excellent clinical response after DBS of the bilateral posterior gyrus rectus rather than the initially targeted CG-25. The remaining four patients with DBS of the CG25 were considered as non-responders. In the case patient, we demonstrate a strong connectivity of the stimulated regions to the medial prefrontal cortex (mPFC), which contrasted to the lower mPFC connectivity in non-responders.

Limitations: confirmation in larger cohorts is needed.

Conclusions: We propose the posterior gyrus rectus as viable alternative new target for DBS in major depressive disorder. High connectivity between target and mPFC supports the pivotal role of this region in brain networks involved in mood processing.

Keywords: deep brain stimulation; major depression disorder; gyrus rectus; subgenual cingulate; medial prefrontal cortex; probabilistic diffusion tractography. 


\section{Introduction}

Currently, there is an ongoing debate about the role of deep brain stimulation (DBS) of the sub-callosal cingulate area (cingulate gyrus (Brodmann Area) 25 - CG-25) in treatment resistant major depressive disorder (MDD). Despite mounting evidence that validates DBS of BA 25 as a safe and successful treatment, a significant number of patients fail to improve clinically (Lozano et al., 2012, 2008). This ambiguity motivates the investigation of the precise mechanisms of CG-25 DBS action in MDD.

Theoretically, successful DBS should modulate dysfunctional limbic circuits while sparing activation of networks supporting other brain functions. In this line of reasoning, recent evidence underscores the fact that the clinical outcome after DBS in MDD is linked to the specificity of activation of particular networks, rather than to the precise anatomical location of implanted electrodes (Johansen-Berg et al., 2008; Lujan et al., 2012; Riva-Posse et al., 2014). DBS responders consistently show activation of projections connecting CG-25 to medial prefrontal cortex (BA 10) and subcortical nuclei, and further propagating to rostral anterior and mid-cingulate cortex. Stimulation of projections to BA 10 was demonstrated to be a strong determinant for prediction of clinical outcome after DBS of the CG-25 (Riva-Posse et al., 2014).

Here, we bring additional evidence for the unique role of the medial prefrontal cortex, nucleus accumbens and ventral caudate for successful DBS treatment of MDD patients. We discuss the mechanisms underlying the marked symptom improvement in a MDD patient after DBS of the posterior gyrus rectus region and link this to the specific anatomical connectivity patterns of the stimulation site assessed with pre-surgical magnetic resonance imaging (MRI).

\section{Methods}

\section{Clinical Assessment}

All patients here described belong to a cohort included in a pilot of a randomized controlled clinical trial (ClinicalTrials.gov NCT00531726) assessing the clinical effects of CG25 stimulation for MDD. Inclusion and exclusion criteria for surgery comply with international standards and have been detailed elsewhere (Merkl et al., 2013). All patients were considered treatment-resistant after failure of complex pharmacotherapy and electroconvulsive therapy (except for patient \#3 who rejected ECT before inclusion in the present study). There were no additional treatment attempts using transcranial magnetic stimulation (TMS) or direct-current stimulation (tDCS). We report a subset of five patients partially overlapping with the previously described (Merkl et al., 2013), with available pre-surgery diffusion weighted imaging (DWI). Upon inclusion, all patients were in a current depressive episode as 
determined by the Structured Clinical Interview for DSM-IV Axis I disorders (First et al., 1996) with a minimum score of 20 on the Hamilton Depression Rating Scale, 24 Items version (HAMD-24, see Table 1, Hamilton, 1960). After electrode implantation, each homologous contact pair was activated separately on five consecutive days followed by antidepressant effects assessment 24 hours later (contact labels: right, ventral to dorsal: 0,1,2,3; left, ventral to dorsal: 4,5,6,7; homologous contacts pairs: 0 and 4; 1 and 5; 2 and 6; 3 and 7). Due to the difficulty of assessing acute effects on depression, stimulation parameters were chosen according to previously published protocols (Holtzheimer et al., 2012; Lozano et al., 2012), and were the same across all contacts and patients tested $(90 \mu \mathrm{s}, 130 \mathrm{~Hz}$, $5 \mathrm{~V}$ ). The patient and the rating clinician were not aware of the stimulation mode (real vs. sham) or of the contact pair chosen for stimulation. After each pair had been active for 24 hours including one sham trial, the best pair was chosen according to the individual decrease of depressive symptoms. All patients had a monthly assessment of clinical improvement including HAMD-24 and Beck Depression Inventory (BDI) scores. Clinical response was defined for a decrease of at least $50 \%$ of both HAMD-24 and BDI. Concomitant medication was held stable for 6 weeks prior to and after surgery.

\section{Neuroimaging}

Before DBS the patients underwent diffusion-weighted imaging (DWI) on a 3T whole-body MRI system (Magnetom TIM Trio, Siemens Healthcare, Erlangen, Germany) using a 32-channel radiofrequency (RF) head receive coil and RF body transmit coil. The DWI protocol was performed with the following parameters: TE $=80 \mathrm{~ms}$, TR $8300 \mathrm{~ms}$, acquisition matrix $128 \times 128$ voxels, 74 axial slices (voxel size of $1.7 \times 1.7 \times 1.7 \mathrm{~mm}$ ), diffusion weighting at a high $\mathrm{b}=1000 \mathrm{~s} \mathrm{~mm}-2$ along 60 directions. Additional structural MRI was acquired in the same session and included a 3D-FLASH magnetization transfer weighting contrast (MTw; TR/ $\alpha=23.7 \mathrm{~ms} / 6^{\circ}$, Weiskopf et al., 2013).

After DBS all patients except patient \#1 underwent a T2-weighted fast acquisition in a MRI in a 1.5T scanner (NT Intera, Philips, Netherlands, as detailed in Merkl et al., 2013). Patient \#1 had a CT scan after DBS.

Pre- and post- DBS MRI images were intra-individually registered linearly to the diffusion space. The voxels corresponding to the susceptibility artifact on MRI or CT were manually identified for each active contact. Starting from these coordinates, we created cube-shaped seed masks for tractography by expanding to all neighboring voxels (total seed volume $=27$ voxels). Whole-brain unconstrained probabilistic tractography was performed in subject-specific native space using the default settings in FSL bedpost (Behrens et al., 2007) modeling up to two fiber directions per voxel with the following parameters: 10000 originating tracts per voxel, curvature 0.2 , step length 0.5 . Resulting tracts were non-linearly transformed into standard Montreal Neurological Institute - MNI space using spatial 
registration parameters estimated in the "unified segmentation" approach of SPM8 (SPM8; Wellcome Trust Centre for Neuroimaging, London, UK, http://www.fil.ion.ucl.ac.uk/spm) running under Matlab 7 (Mathworks, Sherborn, MA, USA). Finally, the calculated tract counts were converted into their natural logarithm.

\section{Results}

\section{Clinical Response to Surgery}

Patients \#2, \#3 and \#4 correspond to patients \#4, \#5, and \#6 in a recent publication by our group (Merkl et al., 2013), to which we refer for further clinical information. The stimulation parameters were the same in all patients $(5 \mathrm{~V}, 90 \mu \mathrm{s}, 130 \mathrm{~Hz})$. Four out of five patients did not show any significant sustained response to stimulation (Table 1). In non responders, the acute assessment did not show a clear effect of stimulation, but homologous contact pairs showing the best trend were chosen for chronic stimulation. Two patients (patients \#3 and \#4) requested removal of the DBS system before the one year follow-up.

Only one patient $(\# 2)$ reported significant improvement in her mood symptoms, as assessed by both HAMD-24 and BDI scores (Table 2). At the time of enrollment in the study, this female patient was 60 years old. She was first diagnosed with MDD at the age of 20 years, and she suffered from four major depressive episodes and one suicide attempt. After numerous pharmacological and electroconvulsive treatments she was considered treatment-resistant, and accepted to participate to the clinical trial. At the time of enrolment, the HDRS-24 was 28 and the BDI score 34. After uncomplicated DBS surgery, we observed satisfactory clinical outcome with a HDRS reduction of $61 \%$ and BDI score reduction of $65 \%$. Both subjective and objective effectiveness of antidepressant treatment was maintained at month 1, 3 and 6 after surgery (see Table 2). At month 9, an ovarian cancer was diagnosed followed by uterus extirpation (month 10). After this additional stressor, the depressive symptoms showed a trend for worsening. However, we observed an improvement between month 12 and 13 with a total symptom reduction of $89 \%$ (HDRS-24 score of 3) in month 18 after DBS surgery - a state that is still persisting until the last assessment at month 24 (79\% and 73\% scores reduction, respectively, Table 2).

\section{Neuroimaging}

The stimulating contacts in the DBS responder were located within the posterior gyrus rectus bilaterally (Brodmann area 14, according to Ongür and Price, 2000; see Figure 1A). Non-responders stimulating contacts were implanted in the BA 25 as planned (Figure 2). For the connectivity study, we 
averaged tracts originating from activated contacts in non-responder patients. The resulting average map was then subtracted to the tracts originating from the stimulating contacts of the responding patient. The probability of projections reaching MPFC through the minor forceps, nucleus accumbens and anterior caudate through the uncinate fascicle was higher in the responder patient than in the average non-responder. Conversely, we show lower connectivity probability for tracts reaching the anterior and mid-cingulate gyrus through the cingulate bundle in the responder patient (see Figure 1B).

\section{Discussion}

Our results confirm the variability of clinical response after CG-25 DBS in major depressive disorder patients to propose the posterior gyrus rectus region as alternative target. Our neuroimaging results suggest that the therapeutic effects of posterior gyrus rectus DBS were mediated by the modulation of circuits involving mainly the mPFC, hence through stimulation of a different than CG-25 node within the very same cortico-subcortical circuit.

The observed therapeutic impact of DBS through modulation of a distributed anatomical network involved in mood processing (Price and Drevets, 2010) confirms the notion of multiple suitable targets for DBS. Supportive evidence for this assumption comes from partially successful DBS of nucleus accumbens, ventral striatum/ventral capsule, habenulae, inferior thalamic nucleus and the medial forebrain bundle (Morishita et al., 2014). According to our results, the gyrus rectus with its strong reciprocal projections to $\mathrm{MPFC}$ and other limbic structures including anterior cingulate, amygdala, hippocampus and hypothalamus (Carmichael and Price, 1996, 1995; Cavada et al., 2000; Kringelbach and Rolls, 2004; Ongür and Price, 2000) has the potential of alternative DBS target. As part of the medial-prefrontal network, gyrus rectus is the main origin of descending connections to hypothalamus and brainstem to mediate the interaction between prefrontal sensory integration regions and visceromotor centers (Ongür and Price, 2000). As previously suggested, the modulation of projections between CG-25 and mPFC plays a central role in the clinical response to DBS additionally to its strong impact on the cingulate cortex and subcortical nuclei (Riva-Posse et al., 2014). While our responder patient had higher contact connectivity to the anterior caudate, we could not confirm a stronger connectivity to the cingulate cortex in the comparison with non-responders. In line with Riva-Posse et al., we interpret our data as confirmatory evidence that the modulation of the three pathways - mPFC, cingulate and deep brain nuclei, is needed to achieve a sustained clinical response. We further speculate that the DBS induced modulation of the mPFC via CG25 or the gyrus rectus region could represent the core mechanism determining clinical efficacy. 


\section{Limitations}

The main novelty of our findings resides in the location of the stimulating contact in the responding patient. The preoperative acquisition of DWI was not possible for the whole cohort described in (MerkI et al., 2013), and did not allow for sound formal statistics in comparing tractography results among responder and non-responders. The high number of non-responders most likely represents random selection of patients, limits however the generalization of our results. The seed volume - a cube of 27 voxels centering the stimulating contact - is probably a good approximation of the tissue volume activated by DBS, thanks to the high resolution of diffusion sequences (1.7 $\mathrm{mm}$ isotropic). Studies on bigger cohorts would definitely profit from recent DBS activation volume models (Riva-Posse et al., 2014).

Given the relatively low success of CG-25 in our patients where four out of five failed to improve, our tractography results support the hypothesis that individually tailored estimation of DBS target might provide better clinical results (Riva-Posse et al., 2014). Despite the promising aspects of the obtained neuroimaging results we fully acknowledge the necessity to evaluate their impact on clinical decision making about the choice of DBS target in MDD, which we will consider in our future research.

\section{Conclusions}

We bring supportive evidence for the suitability of the posterior gyrus rectus as DBS target for treatment resistant depression. Additional experimental and clinical data is needed to fully understand the interplay among different brain structures involved in mood processing, possibly leading to more effective strategies for MDD DBS, including concurrent stimulation of multiple targets.

\section{Acknowledgments}

The study was supported by the German Research Agency (DFG - Deutsche Forschungsgemeinschaft). Grant Number: KFO 247.

$M B$ received unrestricted grants from Medtronic as well as funding from the DFG and Bundesministerium für Bildung und Forschung.

AK received honoraria from St Jude Medical and Medtronic; travel grants from Ipsen Pharma and Boston Scientific, consultancies from Boston Scientific, and is supported by DFG grant KFO247. G-HS reports having received lecture fees from Medtronic, St. Jude Medical and Boston Scientific. BD is supported by the Swiss National Science Foundation (NCCR Synapsy, project grant $\mathrm{Nr}$ 320030_135679 and SPUM 33CM30_140332/1), Foundation Parkinson Switzerland and Foundation 
Synapsis. The research leading to these results has received funding from the European Union Seventh Framework Programme (FP7/2007-2013) under grant agreement no. 604102 (Human Brain Project). LREN is very grateful to the Roger de Spoelberch and Partridge Foundations for their generous financial support.

\section{Financial Disclosures}

Conflicts of interest: none.

\section{References}

1. Behrens, T.E.J., Berg, H.J., Jbabdi, S., Rushworth, M.F.S., Woolrich, M.W., 2007. Probabilistic diffusion tractography with multiple fibre orientations: What can we gain? Neurolmage 34, 144-155. doi:10.1016/j.neuroimage.2006.09.018

2. Carmichael, S.T., Price, J.L., 1996. Connectional networks within the orbital and medial prefrontal cortex of macaque monkeys. J. Comp. Neurol. 371, 179-207. doi:10.1002/(SICI)1096-9861(19960722)371:2\&It;179::AID-CNE1\&gt;3.0.CO;2-\#

3. Carmichael, S.T., Price, J.L., 1995. Sensory and premotor connections of the orbital and medial prefrontal cortex of macaque monkeys. J. Comp. Neurol. 363, 642-664. doi:10.1002/cne.903630409

4. Cavada, C., Compañy, T., Tejedor, J., Cruz-Rizzolo, R.J., Reinoso-Suárez, F., 2000. The anatomical connections of the macaque monkey orbitofrontal cortex. A review. Cereb. Cortex N. Y. N 1991 10, 220-242.

5. First, M.B., Donovan, S., Frances, A., 1996. Nosology of chronic mood disorders. Psychiatr. Clin. North Am. 19, 29-39.

6. Hamilton, M., 1960. A rating scale for depression. J. Neurol. Neurosurg. Psychiatry 23, 56-62.

7. Johansen-Berg, H., Gutman, D.A., Behrens, T.E.J., Matthews, P.M., Rushworth, M.F.S., Katz, E., Lozano, A.M., Mayberg, H.S., 2008. Anatomical connectivity of the subgenual cingulate region targeted with deep brain stimulation for treatment-resistant depression. Cereb. Cortex N. Y. N 1991 18, 1374-1383. doi:10.1093/cercor/bhm167

8. Kringelbach, M.L., Rolls, E.T., 2004. The functional neuroanatomy of the human orbitofrontal cortex: evidence from neuroimaging and neuropsychology. Prog. Neurobiol. 72, 341-372. doi:10.1016/j.pneurobio.2004.03.006

9. Lozano, A.M., Giacobbe, P., Hamani, C., Rizvi, S.J., Kennedy, S.H., Kolivakis, T.T., Debonnel, G., Sadikot, A.F., Lam, R.W., Howard, A.K., Ilcewicz-Klimek, M., Honey, C.R., Mayberg, H.S., 2012. A multicenter pilot study of subcallosal cingulate area deep brain stimulation for treatmentresistant depression. J. Neurosurg. 116, 315-322. doi:10.3171/2011.10.JNS102122

10. Lozano, A.M., Mayberg, H.S., Giacobbe, P., Hamani, C., Craddock, R.C., Kennedy, S.H., 2008. Subcallosal cingulate gyrus deep brain stimulation for treatment-resistant depression. Biol. Psychiatry 64, 461-467. doi:10.1016/j.biopsych.2008.05.034

11. Lujan, J.L., Chaturvedi, A., Malone, D.A., Rezai, A.R., Machado, A.G., Mclntyre, C.C., 2012. Axonal pathways linked to therapeutic and nontherapeutic outcomes during psychiatric deep brain stimulation. Hum. Brain Mapp. 33, 958-968. doi:10.1002/hbm.21262 
12. Merkl, A., Schneider, G.-H., Schönecker, T., Aust, S., Kühl, K.-P., Kupsch, A., Kühn, A.A., Bajbouj, M., 2013. Antidepressant effects after short-term and chronic stimulation of the subgenual cingulate gyrus in treatment-resistant depression. Exp. Neurol. 249, 160-168. doi:10.1016/j.expneurol.2013.08.017

13. Morishita, T., Fayad, S.M., Higuchi, M.-A., Nestor, K.A., Foote, K.D., 2014. Deep Brain Stimulation for Treatment-resistant Depression: Systematic Review of Clinical Outcomes. Neurother. J. Am. Soc. Exp. Neurother. doi:10.1007/s13311-014-0282-1

14. Ongür, D., Price, J.L., 2000. The organization of networks within the orbital and medial prefrontal cortex of rats, monkeys and humans. Cereb. Cortex N. Y. N 1991 10, $206-219$.

15. Price, J.L., Drevets, W.C., 2010. Neurocircuitry of mood disorders. Neuropsychopharmacol. Off. Publ. Am. Coll. Neuropsychopharmacol. 35, 192-216. doi:10.1038/npp.2009.104

16. Riva-Posse, P., Choi, K.S., Holtzheimer, P.E., Mclntyre, C.C., Gross, R.E., Chaturvedi, A., Crowell, A.L., Garlow, S.J., Rajendra, J.K., Mayberg, H.S., 2014. Defining Critical White Matter Pathways Mediating Successful Subcallosal Cingulate Deep Brain Stimulation for Treatment-Resistant Depression. Biol. Psychiatry. doi:10.1016/j.biopsych.2014.03.029

17. Weiskopf, N., Suckling, J., Williams, G., Correia, M.M., Inkster, B., Tait, R., Ooi, C., Bullmore, E.T., Lutti, A., 2013. Quantitative multi-parameter mapping of R1, PD*, MT, and R2* at 3T: a multi-center validation. Front. Brain Imaging Methods 7, 95. doi:10.3389/fnins.2013.00095 


\section{Captions to Figures}

\section{Figure 1}

A. Postoperative T2-weighted image of DBS responder in axial (left panel), sagittal (center), and coronal (right) planes. The active macro-electrode contacts chosen for chronic stimulation are indicated by a yellow arrow and are located within the posterior gyrus rectus (Brodmann's Area BA 14).

B. Probabilistic tractography results with seeds located in the area of stimulating contacts. Average tract counts from a group of non-responders $(n=4)$ are compared with tract counts in the DBS responder. Higher connectivity (yellow) to medial prefrontal areas (BA 10) and ventral caudate next to lower connectivity (blue) to middle and posterior cingulate in the DBS responder compared with non-responders. Values are expressed as natural logarithms of tract counts (maximum $=10000$ ).

\section{Figure 2}

MNI localization of stimulating contacts for the 5 reported patients. Each contact is projected on a sagittal plane (yellow lines on the axial image). First row: left side, from lateral to medial; second row: right side, from medial to lateral direction. The right bottom panel illustrates the boundaries of BA 25 (subcallosal cingulate area) and BA 14 (gyrus rectus) - adapted from Kringelbach and Rolls, 2004, with permission.

\section{Figure 1S}

Single subject tractography results from active contacts in the proximity of gyrus rectus. Two nonresponders were stimulated from ventral contacts, lying between CG25 and gyrus rectus (left active contact in patient \#1 and right one in patient \#3). 


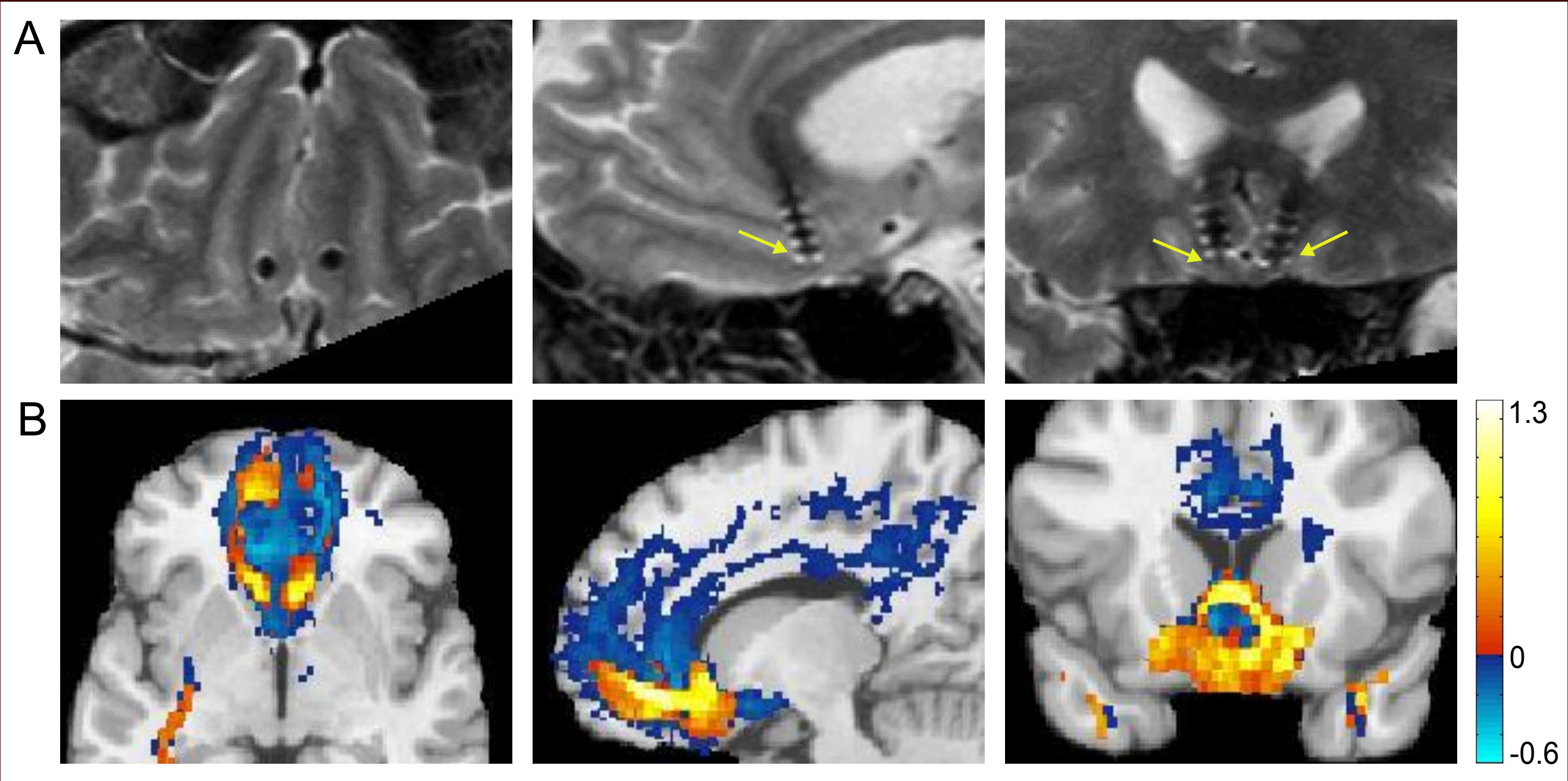




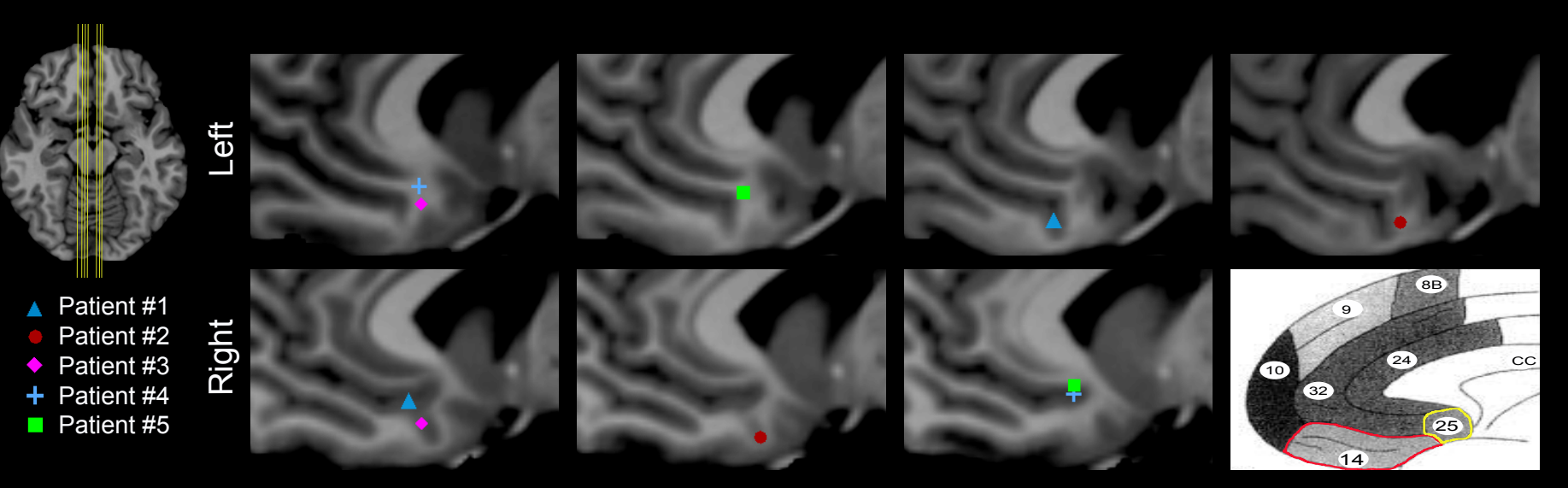


Table 1. Clinical data before and after surgery.

Patient \#2 is the only one showing a sustained clinical benefit. Note that medication was held stable 6 weeks prior and 6 months after the operation. Patients \#3 and \#4 requested the removal of the DBS system before the one year follow-up. ATHF= Antidepressant Treatment History Form. Higher scores indicate higher resistance; $D E=$ Depressive Episodes; $E C T=$ electroconvulsive therapy; HAMD24=Hamilton Depression Rating Scale 24-item version; BDI=Beck Depression Inventory.

\begin{tabular}{|c|c|c|c|c|c|c|}
\hline Patient & & 1 & 2 & 3 & 4 & 5 \\
\hline Age/sex & & $55 / \mathrm{m}$ & $60 / f$ & $36 / \mathrm{m}$ & $50 / m$ & $25 / m$ \\
\hline Age at onset & & 35 & 20 & 16 & 34 & 20 \\
\hline ATHF & & n.a. & 14 & 17 & 20 & 20 \\
\hline$D E$ & & 4 & 10 & 5 & 5 & 3 \\
\hline ECT lifetime & & 35 & 32 & 0 & 18 & 22 \\
\hline \multirow[t]{3}{*}{ HAMD-24 } & Preop. & 31 & 28 & 24 & 32 & 28 \\
\hline & $\begin{array}{l}\text { Postop. } \\
3 \text { months }\end{array}$ & 29 & 16 & 31 & 36 & 26 \\
\hline & $\begin{array}{l}\text { Postop. } \\
6 \text { months }\end{array}$ & 21 & 7 & 33 & 29 & 33 \\
\hline \multirow[t]{3}{*}{$B D I$} & Preop. & 43 & 34 & 57 & 41 & 30 \\
\hline & $\begin{array}{l}\text { Postop } \\
3 \text { months }\end{array}$ & 31 & 17 & 52 & 37 & 37 \\
\hline & $\begin{array}{l}\text { Postop. } \\
6 \text { months }\end{array}$ & n.a. & 12 & 52 & 42 & 33 \\
\hline Medication & & $\begin{array}{l}\text { Pregabaline, } \\
\text { agomelatine, } \\
\text { quetiapine, } \\
\text { levothyroxine }\end{array}$ & $\begin{array}{c}\text { Lithium } \\
\text { tranycypromi } \\
\text { ne } \\
\text { pregabaline } \\
\text { quetiapine }\end{array}$ & $\begin{array}{c}\text { No } \\
\text { medication }\end{array}$ & $\begin{array}{l}\text { Zopiclone, } \\
\text { quetiapine, } \\
\text { trimipramine }\end{array}$ & Mirtazapin \\
\hline
\end{tabular}


Table 2. Clinical assessment of the responding patient (\#2).

Sustained benefit was observed up to the two years assessment. HAMD-24=Hamilton Depression Rating Scale 24-item version; BDI=Beck Depression Inventory. Note a transitory worsening of depressive symptoms at month 12 , corresponding to the diagnosis of an ovary cancer followed by uterus extirpation. Therefore, an additional rating (month 13) was obtained.

\begin{tabular}{lcccc}
\hline & HAMD-24 & $\begin{array}{c}\text { Change of HAMD-24 } \\
\text { (compared to baseline) }\end{array}$ & BDI & $\begin{array}{c}\text { Change of BDI } \\
\text { (compared to baseline) }\end{array}$ \\
\hline Preop. & 28 & 0 & 34 & 0 \\
\hline Postop. & 11 & $-61 \%$ & 12 & $-65 \%$ \\
Month 1 & 6 & $-79 \%$ & 13 & $-62 \%$ \\
Month 3 & 16 & $-43 \%$ & 17 & $-50 \%$ \\
Month 6 & 7 & $-75 \%$ & 12 & $-65 \%$ \\
Month 9 & 20 & $-29 \%$ & 19 & $-44 \%$ \\
Month 12 & 23 & $-18 \%$ & 37 & $+9 \%$ \\
Month 13 & 10 & $-64 \%$ & 11 & $-68 \%$ \\
Month 15 & 2 & $-93 \%$ & 3 & $-91 \%$ \\
Month 18 & 3 & $-89 \%$ & 5 & $-85 \%$ \\
Month 24 & 6 & $-79 \%$ & 9 & $-73 \%$ \\
\hline
\end{tabular}

